\title{
ISSUES IN MEDICINE Will clinical associates be effective for South Africa?
}

\author{
Jane Doherty, Ian Couper, Sharon Fonn
}

South Africa has developed an innovative mid-level medical worker model that can contribute substantively to the development of quality district-level health care. These clinical associates entered the South African job market in 2011 and have reportedly been received favourably. The first cohorts performed well on local and national examinations, with pass rates $>95 \%$. They have demonstrated confidence and competence in the common procedures and conditions encountered in district hospitals; reportedly fitted in well at most of the sites where they commenced working; and made a significant contribution to the health team, resulting in a demand for more clinical associates. Universities and provinces involved in producing clinical associates are enthusiastic and committed. However, priorities are to establish sustainable funding sources for training and deployment, provide adequate supervision and support, monitor the initial impact of the new cadre on health services, and manage the sensitivities of the medical and nursing professions around scopes of practice and post levels. Longer-term concerns are national leadership and support, scaling up of training, the development of career pathways, and the improvement of working conditions at district hospitals.

S Afr Med J 2012;102(11):833-835. DOI:10.7196/SAMJ.5960
The Green Paper on national health insurance (NHI) laid out the South African government's vision for achieving universal health coverage. International evidence indicates that universal coverage requires wellfunctioning district-level services that include the most disadvantaged and remote communities. ${ }^{1}$ Staffing such services with sufficient numbers of appropriately trained health professionals is a major challenge globally and a prerequisite for successful implementation of NHI in South Africa. The Human Resources for Health Strategy ${ }^{2}$ recognises this and commits the country to developing mid-level health workers as one of several approaches to address human resource shortfalls.

New categories of mid-level health workers have been trained and deployed over the past decade, albeit in very small numbers. Conflicts over their scopes of practice, and the brain drain of new graduates into private practice, have weakened their impact on the public sector. We reflect on whether the latest category, a mid-level medical worker or clinical associate, has the potential to substantially strengthen the district health system.

Walter Sisulu University began training its first cohort of 23 clinical associate students in 2008, followed by Pretoria and Wits universities (55 and 25 students respectively). The Walter Sisulu University students graduated in 2010 and were employed in the public health sector at the beginning of 2011, followed by the Wits and Pretoria cohorts in 2012.

\section{Key features of the clinical associate concept}

The clinical associate concept was developed on the basis of international experience of similar cadres (in Kenya, Malawi, Mozambique, Netherlands, Tanzania and the USA) as well as South

Jane Doherty is an independent researcher and part-time lecturer at the School of Public Health, University of the Witwatersrand; Ian Couper is Head of the Division of Rural Health, University of the Witwatersrand; and Sharon Fonn is Professor at the School of Public Health, University of the Witwatersrand.
African experience of other mid-level workers., ${ }^{3,4}$ Policymakers also took account of the sensitivities of other professional groups through a series of consultations to define the scope of practice and service points in which this new cadre would be deployed. As a result, South Africa has a distinctive version of a mid-level medical worker:

1. The clinical associate is conceptualised as a health professional providing a long-term solution to human resource constraints in district hospitals. Their scope of practise is tailored to the specific context and needs of the district hospital, avoiding overlap with primary healthcare nurses who function at clinic and health centre level. The clinical associate is therefore an integral member of a collaborative district-level clinical team that includes doctors and primary healthcare nurses.

2. Clinical associates may only practise under the supervision of doctors with whom they work closely. They assist doctors in providing emergency care, performing diagnostic and therapeutic procedures and in-patient care, and are also expected to have consultation, physical examination and counselling skills. It is intended that this will ensure adequate supervision and support and, again, reduce conflicts around scopes of practice.

3. Training emphasises generalist skills (because of the heavy generalist workload at the district hospital level) and flexibility in response to local needs. Clinical associates are envisaged to develop some specialised skills under the tutelage of doctors with whom they work, depending on the particular needs of the hospital, interests of the local doctors and the capabilities of the individual clinical associate. Specialised postgraduate courses will be developed over time.

4. Training is standardised through a national curriculum that determines the broad skills and learning outcomes that must be achieved, but allows participating universities some flexibility in their courses. To foster close synergy between clinical associates and doctors and confer status on the new cadre, the three-year degree course is offered by medical schools and they are regulated by the Medical and Dental Board. Students must pass a national examination to ensure equivalent standard training.

5. The course is predominantly service-based with most training happening at designated district hospitals. This is because of international evidence for this approach to medical 


\section{FORUM}

education, ${ }^{5,6}$ to ensure that students graduate with extensive practical experience and knowledge of local circumstances, and to demonstrate students' usefulness to other staff by immediately relieving the workload of doctors and nurses.

6. Most students are recruited from districts close to where they train. The Eastern Cape, Gauteng and North West provincial Departments of Health have been instrumental in advertising, selecting students, awarding bursaries, assisting in refurbishing training sites, and creating joint provincialacademic posts which are critical to attract good staff and sustain the quality of training at district hospitals.

Together these features attempt to realise the potential benefits identified in Table 1

\section{Ongoing challenges}

South Africa has developed an innovative clinical associate model with the potential to contribute substantively to the development of quality district-level healthcare. The development of district-level training platforms that encourage multidisciplinary collaboration also opens up the possibility of training other health professionals intended to work at the district level - including nurse clinicians, doctors and family physicians - in close contact with the communities they serve, with an emphasis on team-work and realising the primary healthcare approach.

However, the international literature suggests that mid-level worker programmes fail not so much as a result of the way they have been conceived but rather as a result of '... weaknesses relating to poor teamwork, competing market forces, [poor] production processes and employment opportunities as well as lack of synergy between individual role players and the processes of regulation, production and employment. ${ }^{16}$ Concerns around these issues have been raised in the South African context (Table 1). ${ }^{3,7}$

Therefore, policy-makers and planners should address the following critical issues to ensure that the potential of clinical associates is realised. Firstly, while vacant posts and existing budgets are currently being used to employ new clinical associates in the public sector, government has a moral imperative to establish and fund new posts to accommodate new graduates, otherwise they will have to seek employment in the private sector. Career paths also must be mapped out to prevent a brain drain once new graduates have worked back their bursaries. Their position and role relative to other clinical staff must be better clarified. Clinical associates will be as affected by poor working conditions as any other cadre, hence the overall strengthening of district health services is an important strategy for retention.

Secondly, clinical associates are dependent on the presence of supervising doctors to function as intended. Past problems with providing adequate supervision for community service doctors highlight the importance of appointing doctors along with clinical associates, especially in disadvantaged communities. Invariably there will be pressures on clinical associates to take on more and more of the duties normally associated with doctors, especially in underserved rural areas, as has been the experience in other countries. Rapid assessments of the early effects and experiences of clinical associates would be useful to inform future planning and to monitor the quality of care provided by them.

Thirdly, clinical associates are unlikely to have a meaningful influence unless they are present in significant numbers. 
Table 1. Perceived benefits of, and concerns regarding, clinical associates ${ }^{3}$

\section{Benefits}

They will perform tasks that nurses currently perform because there is no-one else to do them but which are outside their scope of practice, allowing nurses to fulfil their own roles better and become more effective.

They will perform tasks and procedures that have been delegated by doctors, allowing doctors to focus on tasks for which they are uniquely qualified e.g. diagnostic work and supporting the development of primary care services.

They will strengthen district-level health services, allowing different professionals to provide better quality care within their own scopes of practice, improving access for marginalised communities and reducing the need for referral.

They are less costly to train than doctors because their course is shorter.

They will spur the development of district hospital training sites that can be used for training several types of health professional in a multidisciplinary setting, also allowing development of teaching approaches and materials that benefit students from other professions.

They represent a new means of entry for matriculants into the medical field, providing training access in particular for people from marginalised communities.

Because they derive from the local community there will be no language barrier, and they will be more culturally sensitive, better health educators and more successful change agents in their place of work.

They will be easier to retain in the public sector service because they will derive from, and work in, remote communities and have a strong commitment to these communities. There will be less brain drain overseas because their degree is not recognised internationally.

\section{Concerns}

They could become a second-best option for poor and rural communities in response to the brain drain of doctors to the private sector and overseas, and as a way to cut costs, without regard to the effect on quality of care.

They could become a device to avoid having to address the ongoing human resource concerns of doctors and nurses.

They could have an unsatisfactory relationship with doctors and nurses, especially if lines of accountability and post levels are not sorted out appropriately, as this new relationship is not yet well defined.

They could end up working without appropriate supervision because of doctor shortages, as sometimes happens with community service doctors, which could compromise the quality of care.

They could potentially be exploited in the private sector by performing activities for which the doctor will charge and be paid.

Production must be scaled up substantially to reach the minimum target of 1350 clinical associates, a figure based on the target of five clinical associates per district hospital. ${ }^{2}$ This requires a substantial allocation of funds from central government, and the development of new district hospital training sites and strategies to maintain the quality of training in a period of rapid expansion.

Fourthly, regulatory issues are likely to be an ongoing challenge - as they continue to be more than 40 years after the introduction of physician assistants in the USA. Prescribing rights have already emerged as an issue, while some health professionals are uncomfortable with the competency-based scope of practice and want a more narrowly defined job description. Health professionals who feel threatened by clinical associates are likely to attempt to use regulatory issues to undermine their work or curtail their usefulness.

\section{Conclusion}

Participating universities and their partnering provinces are enthusiastic about the concept and reality of clinical associates. Health professionals at training sites have reportedly received the new cadre favourably. The first cohorts of students have performed well on local and national examinations, with pass rates $>95 \%$, and have demonstrated confidence and competence in the common procedures and conditions encountered in district hospitals. Anecdotal accounts suggest that qualified clinical associates have fitted in well at most of the district hospital sites where they have commenced working. They have made a significant contribution to the work of the health team, resulting in a demand for more of these health workers. However, formal evaluations are required to verify these early positive impressions; until then, it is too soon to judge whether this new cadre provides a workable solution to human resource shortages at district hospitals.
The sustainability of existing training models is a concern if student numbers are increased dramatically, as is the absorptive capacity of the public sector, given past problems with employing new categories of mid-level worker and providing adequate supervision to community service graduates. While universities and their partnering provincial Departments of Health are responsible for the daily implementation of the clinical associate programme, strong national leadership is required to provide support and co-ordination, ensure regulatory issues are addressed speedily, and make critical decisions about training funding. This includes managing the sensitivities of the nursing, pharmacy and medical professions, especially around scopes of practice and post levels. Above all, attention must be paid to making the public sector an employer of choice. This is pivotal to retaining clinical associates in the public sector and the profession, as well as to the implementation of NHI.

Gilson L, Doherty J, Loewenson R, Francis V. Challenging inequity through health systems. Healt Systems Knowledge Network, World Health Organisation Commission on the Social Determinants Health. Johannesburg: Centre for Health Policy, EQUINET, London School of Hygiene and Tropical Medicine 2008;48-52.

National Department of Health. Human Resources for Health, South Africa. HRH Strategy for the Health Sector: 2012/13-2016/17. Pretoria: National Department of Health, 2011:74, 99.

3. Doherty J, Conco DN. Mid-level medical workers in South Africa: a situation analysis. Johannesburg School of Public Health, University of the Witwatersrand, 2009:5-6.

Wilson NW, Couper ID, De Vries E Reid S. Fish T, Marais BJ. A critical review of interventions to

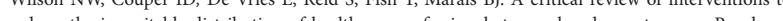
redress the inequitable distribution of healthcare professionals to rural and remote areas. Rural and Remote Health 2009,9 (online).1060. http.//www.rth.org.au (accessed 5 March 2012).

World Health Organization. In reasing Access to Health Workers in Remote and Rural Areas throug mproved Retention. Global Polcy Recommindations. Geneva. World Health Organization, 2010. http://whqlibdoc.who.int/publications/2010/9789241564014_eng.pdf (accessed 5 March 2012).

6. Hugo J, Tshabalala Z, Couper I, et al. Midlevel Medical Worker Programme for South Africa Curriculum and Training Plan. Report to National Department of Health. Pretoria: Family Medicin

van Niekerk J. Mid-level workers: high-level bungling? S Afr Med J 2006;96(12):1209-1211. 